\title{
THE OSELEDEC AND SACKER-SELL SPECTRA FOR ALMOST PERIODIC LINEAR SYSTEMS: AN EXAMPLE
}

\author{
RUSSELL A. JOHNSON
}

\begin{abstract}
We give an example illustrating the relation between the Oseledec spectrum (roughly speaking, the set of Lyapunov exponents) and the SackerSell (or continuous) spectrum for Bohr almost periodic linear systems.
\end{abstract}

1. Introduction. The purpose of this note is to illustrate by means of an example the relation between the Oseledec (or measurable) spectrum [10] and the Sacker-Sell (or continuous) spectrum $[\mathbf{1 2}, \mathbf{1 3}]$ for Bohr almost periodic linear systems

$$
x^{\prime}=A(t) x \quad\left(x \in \mathbf{R}^{k}\right) .
$$

Our example complements a result of [7], according to which an endpoint of an interval in the continuous spectrum is necessarily in the measurable spectrum. In fact, equation (1) which we construct has a point in its measurable spectrum which is an interior point of an interval in the continuous spectrum. In itself this property is of no great significance; however, our equation enjoys an additional property of "irreducibility": if $x_{1}(t), x_{2}(t)$ are two nonzero solutions of $(1)$, then there is a sequence $\left\{t_{n}\right\}$ such that the angle $\theta_{n}$ between $x_{1}\left(t_{n}\right)$ and $x_{2}\left(t_{n}\right)$ tends to zero or $\pi$ as $n \rightarrow \infty$.

Let us explain some of the terminology just used. One usually defines Bohr almost periodicity using translation numbers, but it is convenient here to adopt another starting point. Thus let $C$ be the space of bounded, uniformly continuous maps $B$ from $\mathbf{R}$ to $M_{k}(\mathbf{R})=$ set of $k \times k$ real matrices. Give $C$ the topology of uniform convergence on all of $\mathbf{R}$. Define the translation $\tau_{t}: C \rightarrow C:\left(\tau_{t} B\right)(s)=$ $B(t+s)(B \in C ; t, s \in \mathbf{R})$. If $B \in \mathcal{C}$, define the hull $Y=Y_{B}$ of $B$ to be $\operatorname{cls}\left\{\tau_{t}(B) \mid t \in\right.$ $\mathbf{R}$ \}. We say that $B$ is Bohr almost periodic (a.p.) if $Y$ is compact. It turns out that, in this case, $Y$ may be given the structure of a compact, abelian topological group with identity $B$ and multiplication $*$ satisfying $\tau_{t}(B) * \tau_{s}(B)=\tau_{t+s}(B)$ for all $s, t \in \mathbf{R}$. Thus the map $t \rightarrow \tau_{t}(B)$ defines a dense imbedding of the additive group $(\mathbf{R},+)$ in $(Y, *)$.

Suppose now that $A \in C$ is a.p., with hull $Y$. Let $\mu$ be normalized Haar measure on $Y$. Consider the equations

$$
x^{\prime}=\tilde{A}(t) x \quad\left(\tilde{A} \in Y, x \in \mathbf{R}^{k}\right) .
$$

The Oseledec theorem [10] tells us, among other things, that there is a set $\Sigma_{m}=$ $\left\{\beta_{1}, \ldots, \beta_{k}\right\}$ of real numbers with $\beta_{1} \leq \beta_{2} \leq \cdots \leq \beta_{k}$ such that, for $\mu$-a.a. $\tilde{A} \in$ $Y$, the equation ( $(\tilde{1})$ has linearly independent solutions $x_{1}(t), \ldots, x_{k}(t)$ satisfying

Received by the editors December 5, 1985.

1980 Mathematics Subject Classification (1985 Revision). Primary 34C35, 54H20.

Key words and phrases. Almost periodic, minimal set, Lyapunov number. 
$\lim _{t \rightarrow \infty}, \lim _{t \rightarrow-\infty} t^{-1} \ln \left\|x_{k}(t)\right\|=\beta_{k}$. Also, $\beta_{1}+\cdots+\beta_{k}=\lim _{t \rightarrow \infty} t^{-1} \int_{0}^{t} \operatorname{tr} A(s) d s$. In addition, if $\tilde{\Phi}(t)$ is the fundamental matrix solution of $(\tilde{1})$ satisfying $\tilde{\Phi}(0)=I$, then

$$
\lim _{t \rightarrow \infty} t^{-1} \ln \|\tilde{\Phi}(t)\|=\beta_{k}, \quad \lim _{t \rightarrow-\infty} t^{-1} \ln \|\tilde{\Phi}(t)\|=\beta_{1} .
$$

The $\beta_{i}$ 's are called Lyapunov numbers, and are independent of $\tilde{A}$ for $\mu$-a.a. $\tilde{A} \in Y$. The set $\Sigma_{m}$ is the Oseledec or measurable spectrum of $A$.

Next we consider the Sacker-Sell or continuous spectrum $\Sigma_{c}$ of $A$. Recall first that an equation

$$
x^{\prime}=B(t) x \quad\left(x \in \mathbf{R}^{k}\right)
$$

has exponential dichotomy if there exist constants $K>0, \alpha>0$, and a projection $Q: \mathbf{R}^{k} \rightarrow \mathbf{R}^{k}$ such that, if $\Psi(t)$ is the fundamental matrix solution of (2) satisfying $\Psi(0)=I$, then

$$
\begin{array}{ll}
\left\|\Psi(t) Q \Psi^{-1}(s)\right\| \leq K e^{-\alpha(t-s)} & (t \geq s), \\
\left\|\Psi(t)(I-Q) \Psi^{-1}(s)\right\| \leq K e^{-\alpha(s-t)} & (t \leq s)
\end{array}
$$

(see, e.g., Coppel [1] ). Define $\Sigma_{c}=\left\{\lambda \in \mathbf{R} \mid x^{\prime}=(A(t)-\lambda I) x\right.$ does not have exponential dichotomy $\}$ (see $[\mathbf{1 2}, \mathbf{1 3}]$ ). It is known that $\Sigma_{c}$ is a finite union of (at most $k$ ) compact intervals, and that, for any $\tilde{A} \in Y$ and any nonzero solution $x(t)$ of $(\tilde{1})$,

$$
\varlimsup_{t \rightarrow \infty}, \varlimsup_{t \rightarrow-\infty} t^{-1} \ln \|x(t)\| \text { and } \varliminf_{t \rightarrow \infty}^{\varliminf_{i \rightarrow \infty}}, \varliminf_{t \rightarrow-\infty} t^{-1} \ln \|x(t)\|
$$

all belong to $\Sigma_{c}$. That is, all upper and lower Lyapunov numbers are in $\Sigma_{c}$.

Clearly it is of interest to compare $\Sigma_{m}$ and $\Sigma_{c}$. As we have already noted, an endpoint of an interval in $\Sigma_{c}$ is in $\Sigma_{m}$ [7]. For $2 \times 2$ systems (1), Millionshchikov [8] showed that $\Sigma_{m}$ is exactly the set of endpoints of intervals in $\Sigma_{c}$. This is no longer true for three-dimensional systems; in fact we may take

$$
A(t)=\left(\begin{array}{cc}
A_{1}(t) & 0 \\
0 & 0
\end{array}\right),
$$

where $A_{1}(t)$ is $2 \times 2$ with $\Sigma_{c}\left(A_{1}\right)=[-\gamma, \gamma]$, and $\gamma>0[\mathbf{9}]$. Then clearly $\left\{\beta_{1}, \beta_{2}, \beta_{3}\right\}$ $=\{-\gamma, 0, \gamma\}$. However, if $A(t)$ is not reducible to a system in block-form, then the situation is less clear, and one might conjecture that, if (1) is (in some sense) irreducible, then $\Sigma_{m}$ is the set of endpoints of intervals in $\Sigma_{c}$.

The example we will construct has the property that $\Sigma_{c}=\left[b_{1}, b_{2}\right]$ but $\Sigma_{m}=$ $\left\{b_{1}, a, b_{2}\right\}$ with $b_{1}<a<b_{2}$. To explain just how irreducible the example is, we introduce the corresponding projective flow. Thus let $\mathbf{P}^{2}(\mathbf{R})$ be the projective space of all lines $l$ through the origin in $R^{3}$. Let $\mathbf{P}=Y \times \mathbf{P}^{2}(\mathbf{R})$. Define

$$
\hat{\tau}_{t}(\tilde{A}, l)=\left(\tau_{t}(\tilde{A}), \tilde{\Phi}(t) l\right) \quad(t \in \mathbf{R})
$$

where $\tilde{\Phi}(t) l$ is the image of $l$ under $\tilde{\Phi}(t)\left(l \in \mathbf{P}^{2}(\mathbf{R}), \tilde{A} \in Y\right)$. Then $\left\{\hat{\tau}_{t} \mid t \in \mathbf{R}\right\}$ defines a flow $[\mathbf{3}]$ on $\mathbf{P}$. It turns out that $\mathbf{P}$ is a proximal extension of $Y$ : if $l_{1}, l_{2} \in \mathbf{P}^{2}(\mathbf{R})$ and $\tilde{A} \in Y$, then there is a sequence $\left\{t_{n}\right\}$ such that distance $\left(\tilde{\Phi}\left(t_{n}\right) l_{1}, \tilde{\Phi}\left(t_{n}\right) l_{2}\right) \rightarrow 0$ as $n \rightarrow \infty$. This is the property referred to in the first paragraph. More is true: $\mathbf{P}$ contains a unique minimal set $[\mathbf{3}] M$, which is an 
almost-automorphic extension of $Y[\mathbf{5}, \mathbf{1 4}]$. This means that, for $y$ in a residual subset $Y_{0} \subset Y$, the fiber $M_{y}=M \cap\left(\{y\} \times \mathbf{P}^{2}(\mathbf{R})\right)$ reduces to a single point. Thus our example differs from those constructed in $[\mathbf{4}]$, where $M=\mathbf{P}$, and $\Sigma_{m}$ is a single point.

The moral, then, is that $A$ is trying simultaneously to be nilpotent ( $M$ is almost automorphic) and to have three distinct real eigenvalues $\left(\Sigma_{c}\right.$ consists of three distinct points).

2. The example. The construction to follow is basically an elaboration of one of Millionshchikov [9]. The desired matrix function $A(t)$ will be a uniform limit of $T_{n}$-periodic, continuous matrix functions $A_{n}(t)$, where $T_{n+1}=j_{n} T_{n}$ for whole numbers $j_{n}(n=1,2, \ldots)$. Thus $A(t)$ will be limit-periodic.

First of all, we fix some notation. If $0 \neq x \in \mathbf{R}^{3}$, let $[x]$ be the unit vector $x /\langle x, x\rangle^{1 / 2} \in S^{2}$. Let $\varepsilon_{0}=0$, and let $\left\{\varepsilon_{n} \mid n \geq 1\right\}$ be a decreasing sequence of positive numbers such that $\sum_{n=1}^{\infty} \varepsilon_{n}=1$. We will consider systems

$$
x^{\prime}=A_{n}(t) x \quad\left(x \in \mathbf{R}^{3}\right)
$$

let $\Phi_{n}(t)$ be the fundamental matrix solution of $(1)_{n}$ which satisfies $\Phi_{n}(0)=I$.

Let $A_{1}(t)$ be a continuous matrix function of period $T_{1} \geq 4$ with the following properties:

$$
A_{1}(t)=0 \quad\left(t \in[0,1] \cup\left[T_{1}-2, T_{1}\right]\right)
$$

$$
\operatorname{tr} A_{1}(t) \equiv 0
$$

Suppose furthermore that there are linearly independent unit vectors $u_{1}, v_{1}, w_{1}$ such that

$$
\begin{aligned}
& \Phi_{1}\left(T_{1}\right) u_{1}=\left(\exp -5 T_{1}\right) u_{1}, \\
& \Phi_{1}\left(T_{1}\right) v_{1}=v_{1}, \\
& \Phi_{1}\left(T_{1}\right) w_{1}=\left(\exp 5 T_{1}\right) w_{1},
\end{aligned}
$$

and

$$
\theta\left(u_{1}, v_{1}\right)<\left(\varepsilon_{1}\right)^{2}, \quad \theta\left(u_{1}, w_{1}\right)<\left(\varepsilon_{1}\right)^{2} .
$$

Here $\theta(a, b)$ denotes the angle between the unit vectors $a$ and $b$; we always suppose that $0 \leq \theta(a, b)<\pi$ radians. Finally, assume that

$$
-5.1 \leq \frac{1}{t-s} \ln \left\|\Phi_{1}(t) \Phi_{1}^{-1}(s)\right\| \leq 5.1 \quad(t \neq s) .
$$

Clearly a function $A_{1}(t)$ can be found for which $(3)_{1}-(7)_{1}$ are valid.

Now suppose that $A_{n}$ is a continuous matrix function of period $T_{n} \geq 4$ such that

$$
A_{n}(t)=0 \quad\left(t \in[0,1] \cup\left[T_{n}-2+\sum_{i=1}^{n-1} \varepsilon_{i}, T_{n}\right]\right),
$$

$$
\operatorname{tr} A_{n}(t) \equiv 0
$$


Assume in addition that $u_{n}, v_{n}, w_{n}$ are linearly independent unit vectors such that

$$
\begin{aligned}
& \Phi_{n}\left(T_{n}\right) u_{n}=\left(\exp -\gamma_{n} T_{n}\right) u_{n}, \\
& \Phi_{n}\left(T_{n}\right) v_{n}=v_{n}, \\
& \Phi_{n}\left(T_{n}\right) w_{n}=\left(\exp \gamma_{n} T_{n}\right) w_{n}, \quad \gamma_{n} \geq 5-\sum_{i=1}^{n-1} \varepsilon_{i} ; \\
& \quad \theta\left(u_{n}, v_{n}\right)<\varepsilon_{n}^{2}, \quad \theta\left(u_{n}, w_{n}\right)<\varepsilon_{n}^{2} ; \\
& -5.1 \leq \frac{1}{t-s} \ln \left\|\Phi_{n}(t) \Phi_{n}^{-1}(s)\right\| \leq 5.1 \quad(t \neq s) .
\end{aligned}
$$

Our goal is to find a continuous matrix function $A_{n+1}(t)$ and an integer $j_{n} \geq 1$ such that $A_{n+1}$ has period $T_{n+1}=j_{n} T_{n}$, and such that

$$
\left\|A_{n+1}(t)-A_{n}(t)\right\|<4 \varepsilon_{n} \quad(t \in \mathbf{R}) .
$$

To begin, let $P_{n}$ be the plane in $\mathbf{R}^{3}$ spanned by $u_{n}$ and $w_{n}$. Using a technique of Millionshchikov (to be described shortly), we can choose an integer $J_{1} \geq 1$ such that, if $j \geq J_{1}$, then a rotation $R$ of $\mathbf{R}^{3}$ can be found which preserves $P_{n}$, fixes vectors normal to $P_{n}$, satisfies $\|R-I\|<2 \varepsilon_{n}^{2}$, and has the property that the matrix $H=R \circ \Phi_{n}\left(j T_{n}\right)$ fulfills the following conditions:

There are unit vectors $u_{n+1}, w_{n+1} \in P_{n}$ which are "between" (in the obvious sense) $u_{n}$ and $w_{n}$ such that $H u_{n+1}=\left(\exp -\gamma j T_{n}\right) u_{n+1}$ and $H w_{n+1}=\exp \left(\gamma j T_{n}\right) w_{n+1}$ with $\gamma \geq 5-\sum_{i=1}^{n} \varepsilon_{i}$;

$$
\begin{gathered}
\theta\left(u_{n}, u_{n+1}\right)<\theta\left(u_{n}, w_{n+1}\right)<\frac{1}{3} \varepsilon_{n+1}^{2} \min \left(1, \theta\left(u_{n}, w_{n}\right) / \theta\left(u_{n}, v_{n}\right)\right) \\
\theta\left(u_{n}, w_{n+1}\right)>\frac{1}{6} \varepsilon_{n+1}^{2} \min \left(1, \theta\left(u_{n}, w_{n}\right) / \theta\left(u_{n}, v_{n}\right)\right) .
\end{gathered}
$$

We briefly indicate how $J_{1}, R$, and the vectors $u_{n+1}, w_{n+1}$ may be found. Let $\Gamma(t)$ be the restriction of $\Phi_{n}(t)$ to $P_{n}$. Then $\Gamma\left(T_{n}\right)$ is a linear mapping of $P_{n}$ to itself which has eigenvectors $u_{n}, w_{n}$ with eigenvalues $\delta^{-1}, \delta$ respectively, where $\delta=\exp \gamma_{n} T_{n}$ and $\gamma_{n} \geq 5-\sum_{i=1}^{n-1} \varepsilon_{i}$. Consider unit vectors $x \in P_{n}$ which are between $u_{n}$ and $w_{n}$. If $x \neq u_{n}$, then $\lim _{j \rightarrow \infty}\left[\Gamma\left(j T_{n}\right) \cdot x\right]=W_{n}$; i.e., $x$ is rotated towards $w_{n}$ as $j$ increases. Moreover $\lim _{j \rightarrow \infty}\left(1 / j T_{n}\right) \ln \left\|\Gamma\left(j T_{n}\right) x\right\|=\gamma_{n}$.

Let $\tilde{\theta}_{j}(x)$ be the angle between $u_{n}$ and $\left[\Gamma\left(j T_{n}\right) x\right]$. Then $\tilde{\theta}_{j}\left(u_{n}\right)=0$, and $\lim _{j \rightarrow \infty} \tilde{\theta}_{j}(x)=\theta\left(u_{n}, w_{n}\right)$ if $x \neq u_{n}$. For any $0<r<\theta\left(u_{n}, w_{n}\right)$, let $R$ be the rotation of the plane $P_{n}$ which displaces $w_{n}$ towards $u_{n}$ by $r$ radians. Then for large $j$, there will be exactly two vectors between $u_{n}$ and $w_{n}$ which are eigenvectors of $R \circ \Gamma\left(j T_{n}\right)$. If we choose

$$
\begin{aligned}
& \theta\left(u_{n}, w_{n}\right)-\frac{1}{3} \varepsilon_{n+1}^{2} \min \left(1, \theta\left(u_{n}, w_{n}\right) / \theta\left(u_{n}, v_{n}\right)\right) \\
& \quad<r<\theta\left(u_{n}, w_{n}\right)-\frac{1}{6} \varepsilon_{n+1}^{2} \min \left(1, \theta\left(u_{n}, w_{n}\right) / \theta\left(u_{n}, v_{n}\right)\right)
\end{aligned}
$$

then, for large $j,(9)-(11)$ will hold for the eigenvectors $u_{n+1}, w_{n+1}$ of $R \circ \Gamma\left(j T_{n}\right)$ (see $[9]$, also $[6, \S 5])$. 
Returning to the construction of $A_{n+1}$, fix $j \geq J_{1}$. Let $b$ be a continuous map from $\mathbf{R}$ to the set of antisymmetric, real, $3 \times 3$ matrices such that $b$ vanishes outside of $\left[0, \varepsilon_{n}\right], \sup _{t}\|b(t)\| \leq 4 \varepsilon_{n}$, and such that the $3 \times 3$ matrix solution of

$$
\eta^{\prime}=b(t) \eta, \quad \eta(0)=I,
$$

satisfies $\eta\left(\varepsilon_{n}\right)=R$. Consider the function

$$
B(t)=\left\{\begin{array}{l}
A_{n}(t), \quad 0 \leq t \leq j T_{n}-2+\sum_{i=1}^{n-1} \varepsilon_{i} \\
b\left(t-\left[j T_{n}-2+\sum_{i=1}^{n-1} \varepsilon_{i}\right]\right), \quad j T_{n}-2+\sum_{j=1}^{n-1} \varepsilon_{i} \leq t \leq j T_{n}
\end{array}\right.
$$

we extend $B(t)$ to all of $\mathbf{R}$ by $j T_{n}$-periodicity. Let $\Psi(t)$ be the fundamental matrix solution of $x^{\prime}=B(t) x$ which satisfies $\Psi(0)=I$. Then $\Psi\left(j T_{n}\right)=H=R \circ \Phi_{n}\left(j T_{n}\right)$.

We claim that, if $j$ is sufficiently large, we can set $A_{n+1}(t)=B(t)$ and fulfill all conditions $(3)_{n+1}-(8)_{n+1}$. In fact, $(3)_{n+1},(4)_{n+1},(7)_{n+1}$, and $(8)_{n+1}$ are true for any $j \geq J_{1}$, if $A_{n+1}=B$. Moreover, for $j \geq J_{1}$, the vectors $u_{n+1}$ and $w_{n+1}$ satisfy the corresponding parts of $(5)_{n+1}$ and (6) ${ }_{n+1}$. Using (4) $n$, (9), and Liouville's formula, we see that there is a unit vector $v_{n+1} \in \mathbf{R}^{3}$ such that $H v_{n+1}=v_{n+1}$; i.e., (5) $)_{n+1}$ is completely satisfied. So we need only show that $j$ and $v_{n+1}$ can be chosen in such a way that the first part of $(6)_{n+1}$ holds.

To do so, once again fix $j \geq J_{1}$. Consider the spherical triangle $\Delta_{n}$ with vertices $u_{n}, v_{n}, w_{n}$; thus $\Delta_{n} \subset S^{2}$. The vector $w_{n+1}$ lies on the side $u_{n} w_{n}$ (i.e., arc of the great circle containing $u_{n}$ and $w_{n}$ ) of $\Delta_{n}$. Let $p$ be a general point on the side $u_{n} v_{n}$ of $\Delta_{n}$. Let $\varphi_{p}$ be the measure, in radians, of the spherical angle with vertex $w_{n+1}$ and sides $u_{n} w_{n+1}, u_{n} p$.

Let $\varphi_{p}(t)$ be the measure of the spherical angle with vertex $\left[\Phi_{n}(t) w_{n+1}\right]$ and sides determined by $\left[\Phi_{n}(t) u_{n}\right],\left[\Phi_{n}(t) p\right]$. Note that, if $p \neq u_{n}$, then $\lim _{j \rightarrow \infty} \varphi_{p}\left(j T_{n}\right)=$ $\varphi_{\infty}=$ measure of the angle with sides $u_{n} w_{n}$ and $w_{n} v_{n}$. Hence by $(10)$ and spherical trigonometry, one has that, if $\theta\left(u_{n}, p\right)>\frac{1}{3} \varepsilon_{n+1}^{2}$, then for large $j, \varphi_{p}\left(j T_{n}\right)>\varphi_{p}(0)$. On the other hand, if $\theta\left(u_{n}, p\right) / \theta\left(u_{n}, v_{n}\right)<\theta\left(u_{n}, w_{n+1}\right) / \theta\left(u_{n}, w_{n}\right)$, then for large $j$, $\varphi_{p}\left(j T_{n}\right)<\varphi_{p}(0)$. (Note that, by $(11), \theta\left(u_{n}, w_{n+1}\right) / \theta\left(u_{n}, w_{n}\right) \geq c>0$, where $c$ is independent of $j$.) Thus there exists $J_{2} \geq J_{1}$ such that, if $j \geq J_{2}$, then there is a point $p_{0}$ with $\theta\left(u_{n}, p_{0}\right)<\frac{1}{3} \varepsilon_{n+1}^{2}$ and $\varphi_{p_{0}}\left(j T_{n}\right)=\varphi_{p_{0}}(0)$. In particular, the plane $W_{n}$ spanned by $w_{n+1}$ and $p_{0}$ is invariant under $H=R \circ \Phi_{n}\left(j T_{n}\right)$.

Fix $j \geq J_{2}$. We know that there is a unit vector $v_{n+1}$ such that $H v_{n+1}=v_{n+1}$. We also know that $\theta\left(u_{n}, p_{0}\right)<\frac{1}{3} \varepsilon_{n+1}^{2}$. Hence we can show that the first part of $(6)_{n+1}$ holds by proving that $v_{n+1}$ can be chosen to lie on the arc $\sigma=w_{n+1} p_{0}$. To do so, note that, if $x \in \sigma$ is close to $w_{n+1}$, then $[H x]$ is even closer to $w_{n+1}$. This is because $w_{n+1}$ is an eigenvector corresponding to the largest eigenvalue of $H$. On the other hand, the arc $\{[H x] \mid x \in \sigma\}$ is longer than $\sigma$, hence $\left[H p_{0}\right]$ is further away from $w_{n+1}$ than is $p_{0}$ itself. Thus there is a point $x_{0} \in \sigma$ such that $\left[H x_{0}\right]=x_{0}$, and we can take $v_{n+1}=x_{0}$.

We have shown that, if $j \geq J_{2}$, and if $A_{n+1}(t)=B(t)(t \in \mathbf{R})$, then $(3)_{n+1^{-}}$ $(8)_{n+1}$ are satisfied by the system $(1)_{n+1}$.

It will be convenient to impose a further condition on $j$. Namely, let $U_{n} \subset S^{2}$ be an open set containing the arc $u_{n} v_{n}$ such that diameter $U_{n}<\varepsilon_{n}$ and $w_{n} \notin U_{n}$. 
We can choose $J_{3} \geq J_{2}$ such that, for $j \geq J_{3}$ :

$(12)_{n+1}$ if $\pm x \notin U_{n}$, then either $\theta\left(H x, w_{n+1}\right)<\varepsilon_{n+1}^{2}$ or $\theta\left(H x,-w_{n+1}\right)<\varepsilon_{n+1}^{2}$.

Now fix $j_{n} \geq J_{3}$, and let $T_{n+1}=j_{n} T_{n}, A_{n+1}=B$. By induction, we obtain a sequence of matrices $A_{1}, A_{2}, \ldots$, all periodic with periods $T_{1}, T_{2}=j_{1} T_{1}, T_{3}=j_{2} T_{2}$, etc. Conditions $(3)_{n}-(8)_{n}(n \geq 1)$ and $(12)_{n}(n \geq 2)$ hold for the corresponding systems $(1)_{n}$. Let $A(t)=\lim _{n \rightarrow \infty} A_{n}(t)$, so that $A(t)$ is limit-periodic (see $(8)_{n}$ ). Let $Y$ be the hull of $A$, and consider the equations

$$
x^{\prime}=\tilde{A}(t) x \quad(\tilde{A} \in Y) .
$$

Let $\Sigma_{c}$ be the continuous spectrum of equation (1). We claim that $\Sigma_{c}$ is a single interval. For, if not, there would exist a projection $Q: \mathbf{R}^{3} \rightarrow \mathbf{R}^{3}$ and real numbers $\alpha_{1} \leq \alpha_{2}<\alpha_{3} \leq \alpha_{4}$ such that: (i) $Q \neq 0, Q \neq I$; (ii) if $0 \neq x=Q x$, then $\varlimsup_{\lim } t \rightarrow \pm \infty, \underline{\lim }_{t \rightarrow \pm \infty} t^{-1} \ln \|\Phi(t) x\| \in\left[\alpha_{1}, \beta_{1}\right]$; (iii) if $0 \neq x=(I-Q) x$, then $\varlimsup_{t \rightarrow \pm \infty}, \underline{\lim }_{t \rightarrow \pm \infty} t^{-1} \ln \|\Phi(t) x\| \in\left[\alpha_{2}, \beta_{2}\right]$ (see [12, 13]). Now we use a perturbation theorem of Coppel [1] to conclude that, for large $n$, there are projections $Q_{n} \rightarrow Q$ and constants $\alpha_{i}^{n}(1 \leq i \leq 4)$ such that the above statements hold with $\Phi_{n}(t)$ in place of $\Phi(t)$. It is easily seen, however, that the range of $Q_{n}$ must be a sum of eigenspaces of $\Phi_{n}\left(T_{n}\right)$. Thus $Q_{n} \rightarrow Q$ is inconsistent with (6) $)_{n}$ for large $n$, and we conclude that $\Sigma_{c}$ is indeed a single interval.

Let $\Sigma_{c}=\left[b_{1}, b_{2}\right]$ with $b_{1} \leq b_{2}$. Let $\Sigma_{m}=\left\{\beta_{1}, \beta_{2}, \beta_{3}\right\}\left(\beta_{1} \leq \beta_{2} \leq \beta_{3}\right)$ be the measurable spectrum of equations ( $\tilde{1})$. Then $\beta_{1}=b_{1}, \beta_{3}=b_{2}[\mathbf{7}]$. It follows from $(7)_{n}$ of our construction that $\left\|\Phi(t) \Phi^{-1}(s)\right\| \leq \exp 5.1(t-s)$ for all $t \geq s$. Hence $\ln \left\|\tilde{\Phi}(t) \tilde{\Phi}^{-1}(s)\right\| \leq 5.1(t-s)$ for $t-s \geq 0$, for all $\tilde{A} \in Y$; we use the fact that $\left\{\tau_{t}(A) \mid t \in \mathbf{R}\right\}$ is dense in $Y$. Hence $b_{2} \leq 5.1$. Similarly $b_{1} \geq-5.1$. However, we also know that $b_{2} \geq \varlimsup_{t \rightarrow \infty} t^{-1} \ln \|\tilde{\Phi}(t)\|$ (e.g., $\left.[\mathbf{2}, 7]\right)$. Hence by $(5)_{n}, b_{2} \geq 4$. Similarly $b_{1} \leq-4$. Since $\operatorname{tr} A(t) \equiv 0$, we have $\beta_{1}+\beta_{2}+\beta_{3}=0$. It is now clear that $\beta_{2} \neq \beta_{1}, \beta_{2} \neq \beta_{3}$. Thus $\Sigma_{m}$ consists of three distinct numbers.

Next we consider irreducibility properties of equation (1). We show first that $\mathbf{P}=Y \times \mathbf{P}^{2}(\mathbf{R})$ contains a unique minimal set $M$ which is an almost automorphic extension of the base $Y$. To do so, fix attention on $A \in Y$. Let $\Delta_{n}$ be the spherical triangle with vertices $u, v, w(n \geq 1)$, and let $\left\{x_{0}\right\}=\bigcap_{n=1}^{\infty} \Delta_{n}$. Let $\left\{s_{k}\right\}$ be a sequence such that $\tau_{s_{k}}(A) \rightarrow A$. Using the duality theory of compact, abelian topological groups [11], one can show that, for fixed $n, s_{k} \bmod T_{n} \rightarrow 0$ as $k \rightarrow \infty$. Fix $n$, and choose $K=K(n)$ such that, if $k \geq K$, then $\left|s_{k} \bmod T_{n}\right| \leq \frac{1}{2}$. It follows from our construction that, if $m \geq n$ and $j \geq 0$, then $\left\{\left[\Phi_{m}\left(j T_{n}\right) x\right] \mid x \in \Delta_{m}\right\} \subset \Delta_{n}$ (see $(5)_{n},(9)$, and note that $\Delta_{n+1} \subset \Delta_{n}$ for $n \geq 1$ ). Since $A(t)=A_{m}(t)$ for $0 \leq t \leq T_{m}$, and since $A_{m}(t)=0$ for $T_{m}-1 \leq t \leq T_{m+1}(m \geq 1)$, we see that $\left[\Phi\left(s_{k}\right) x_{0}\right] \in \Delta_{n}$ for $k \geq K$. Projecting from $S^{2}$ to $\mathbf{P}^{2}(\mathbf{R})$, and recalling from $\S 1$ the definition of the flow $\hat{\tau}$ on $\mathbf{P}$, we conclude that $\hat{\tau}_{s_{k}}\left(A, l_{0}\right) \rightarrow\left(A, l_{0}\right)$ as $k \rightarrow \infty$. Here $l_{0} \in \mathbf{P}^{2}(\mathbf{R})$ is the line containing $x_{0}$.

Let $M=\operatorname{cls}\left\{\tau_{t}\left(A, l_{0}\right) \mid t \in \mathbf{R}\right\} \subset \mathbf{P}$. Then $M \cap\left(\{A\} \times \mathbf{P}^{2}(\mathbf{R})\right)$ equals $\left\{\left(A, l_{0}\right)\right\}$, i.e. is a singleton. It follows without difficulty that $M$ is a minimal, almost automorphic extension of $Y$ (see, e.g., [14]).

To prove uniqueness of $M$, suppose that $M_{1} \neq M$ is another minimal subset of P. Then $M_{1} \cap M=\varnothing$. Let $\left(A, l_{1}\right) \in M_{1}$, and let $0 \neq x$ be an element of the line $l_{1}$. Then $[\Phi(t) x]$ is bounded away from $\left[\Phi(t) x_{0}\right]$, uniformly in $t \in \mathbf{R}$. This contradicts $(12)_{n+1}$ and the fact that $A(t)=A_{n}(t)$ for $0 \leq t \leq T_{n}$, when $n$ is taken large. 
Finally, we show that $\mathbf{P}$ is a proximal extension of $Y$. By minimality of $Y$, it is sufficient to show that, if $l_{1}, l_{2} \in \mathbf{P}^{2}(\mathbf{R})$, then there is a sequence $t_{k} \rightarrow \infty$ such that distance $\left(\Phi\left(t_{k}\right) \cdot l_{1}, \Phi\left(t_{k}\right) \cdot l_{2}\right) \rightarrow 0$ as $k \rightarrow \infty$. However, this follows from conditions $(12)_{n+1}$ and the relation $A(t)=A_{n}(t)\left(0 \leq t \leq T_{n}, n \geq 1\right)$. Thus we have shown that (1) has all the properties set out for it in the Introduction.

It is worth noting that, by our construction, the planes $\left\{P_{n}\right\}$ satisfy $P_{1}=P_{2}=$ $P_{3}=\cdots$. If we choose $A_{1}$ in such a way that $P_{1}$ is invariant under $\Phi_{1}(t)$ (as we certainly can do), then $M$ is in fact a subset of $Y \times P_{1}$.

\section{REFERENCES}

1. A. Coppel, Dichotomies and stabilitity theory, Lecture Notes in Math., vol. 629, SpringerVerlag, Berlin and New York, 1978.

2. J. Daletskii and M. Krein, Stability of solutions of differential equations in Banach space, Transl. Math. Monos., vol. 43, Amer. Math. Soc., Providence, R.I., 1974.

3. R. Ellis, Lectures on topological dynamics, Benjamin, Reading, Mass., 1969.

4. R. Ellis and R. Johnson, Topological dynamics and linear differential systems, J. Differential Equations 44 (1982), 21-39.

5. H. Furstenberg, H. Keynes and L. Shapiro, Prime flows in topological dynamics, Israel J. Math. 14 (1973), 26-38.

6. R. Johnson, The recurrent Hills equation, J. Differential Equations 46 (1982), 165-194.

7. R. Johnson, K. Palmer and G. Sell, Ergodic theory of linear dynamical systems, SIAM J. Appl. Math. (to appear).

8. V. Millionshchikov, Metric theory of linear systems of differential equations, Math. USSRSb. 6 (1968), 149-158.

9. __ Proof of the existence of non-irreducible systems of linear differential equations with almost periodic coefficients, J. Differential Equations 4 (1968), 203-205.

10. V. Oseledec, A multiplicative ergodic theorem, Trans. Moscow Math. Soc. 19 (1968), 197231.

11. L. Pontryagin, Topologische Gruppen, Deutsche Ubersetzung, Teubner Verlag, Leipzig, 1957.

12. R. Sacker and G. Sell, Dichotomies and invariant splittings for linear differential systems. I, J. Differential Equations 15 (1974), 429-458.

13. __ A spectral theory for linear differential systems, J. Differential Equations 27 (1978), $320-358$.

14. W. Veech, Almost automorphic functions on groups, Amer. J. Math. 87 (1965), 719-751.

Department of Mathematics, University of Southern California, Los ANGELES, CALIFORNIA 90089 